Enferm Bras 2020;19(4Supl):S34-S41

https://doi.org/10.33233/eb.v19i4.4337

\title{
REVISÃO \\ O impacto do isolamento social na qualidade de vida dos idosos durante a pandemia por COVID-19
}

Marcos Vinicius Sousa Silva*, Jessica de Almeida Rodrigues*, Mylena de Souza Ribas*, Jessica Cristina Santana de Sousa*, Thiálita Rebeca Oliveira de Castro*, Beatriz Andrade dos Santos*, Julliane Messias Cordeiro Sampaio, D.Sc. ${ }^{\star \star}$, Vanessa Alvarenga Pegoraro, M.Sc. ${ }^{\star \star *}$

\section{${ }^{*}$ Estudante de graduação Enfermagem do UNICEUB, ${ }^{* *}$ Professora Titular do Curso de Enfermagem FACES/UNICEUB, ${ }^{* \star *}$ Professora Assistente do Curso de Enfermagem FACES/UNICEUB}

Recebido em 13 de agosto de 2020; aceito em 30 de agosto de 2020.

Correspondência: Vanessa Alvarenga Pegoraro, Curso de Enfermagem FACES/UNICEUB, salas 707/907 Campus Universitário, SEPN Asa Norte, 70790-075 Brasília DF

\author{
Marcos Vinicius Sousa Silva: marcosxt21@gmail.com \\ Jessica de Almeida Rodrigues: jessica.almeida@sempreceub.com \\ Mylena de Souza Ribas: mylena.ribass@gmail.com \\ Jessica Cristina Santana de Sousa: jessika_santana08@hotmail.com \\ Thiálita Rebeca Oliveira de Castro: thialitacastro@gmail.com \\ Beatriz Andrade dos Santos: beatrizandrade280197@gmail.com \\ Julliane Messias Cordeiro Sampaio: julliane.sampaio@ceub.edu.br \\ Vanessa Alvarenga Pegoraro: vanessa.pegoraro@ceub.edu.br
}

\section{Resumo}

Introdução: $\mathrm{Na}$ atualidade vivenciamos a pandemia de uma doença respiratória detectada na cidade de Wuhan, na China, intitulada COVID-19. Os grupos de risco consistem principalmente em idosos, pessoas com patologias crônicas e imunocomprometidos. Como uma das medidas profiláticas, recomenda-se o isolamento social, em especial o grupo de risco. Objetivo: $O$ presente estudo objetivou apresentar o impacto do isolamento social na vida da pessoa idosa na atual pandemia por COVID-19. Métodos: Trata-se de uma revisão narrativa da literatura na base de dados Scielo, Lilacs e Sistema de Publicação Eletrônica de Teses e Dissertações. Resultados: As discussões foram baseadas nos tópicos sobre a qualidade de vida e a senescência, isolamento social dos idosos em tempos de pandemia e as consequências do isolamento social para o idoso. $O$ envelhecimento saudável se faz necessário no atual momento, já que essa população corresponde a maior parcela vulnerável às consequências da contaminação pelo coronavírus. Conclusão: Portanto, são necessárias intervenções quanto a saúde do idoso no período pandêmico, fornecendo opções da continuidade da qualidade de vida.

Palavras-chave: isolamento social, qualidade de vida, idoso, pandemia, impacto social.

\begin{abstract}
The impact of social isolation on the quality of life of the elderly during the COVID-19 pandemic

Introduction: Currently we are experiencing the pandemic of a respiratory disease detected in the city of Wuhan, China, entitled COVID-19. The risk groups consist mainly of the elderly, people with chronic pathologies and immunocompromised. As one of the prophylactic measures, social isolation is recommended, especially the risk group. Objective: The present study aimed to present the impact of social isolation on the life of the elderly in the current pandemic by COVID19. Methods: This is a narrative review of the literature in the database Scielo, Lilacs and the Electronic Publication System of Theses and Dissertations. Results: The discussions were based on topics on quality of life and senescence, social isolation of the elderly in times of pandemic and the consequences of social isolation for the elderly. Healthy aging is necessary at the present time, as this population corresponds to the largest portion vulnerable to the consequences of contamination by the coronavirus. Conclusion: Therefore, interventions regarding the health of
\end{abstract}


the elderly in the pandemic period are necessary, providing options for the continuity of quality of life.

Keywords: social isolation, quality of life, elderly, pandemics, social impact.

\section{Resumen \\ El impacto del aislamiento social en la calidad de vida de los ancianos durante la pandemia por COVID-19}

Introducción: Actualmente estamos viviendo la pandemia de una enfermedad respiratoria detectada en la ciudad de Wuhan, China, titulada COVID-19. Los grupos de riesgo están formados principalmente por adultos mayores, personas con patologías crónicas e inmunodeprimidos. Como una de las medidas profilácticas, se recomienda el aislamiento social, especialmente el grupo de riesgo. Objetivo: El presente estudio tuvo como objetivo presentar el impacto del aislamiento social en la vida de los adultos mayores en la actual pandemia por COVID-19. Métodos: Se trata de una revisión narrativa de la literatura en la base de datos Scielo, Lilacs y el Sistema de Publicación Electrónica de Tesis y Disertaciones. Resultados: Las discusiones se basaron en temas sobre calidad de vida y senescencia, aislamiento social de los mayores en tiempos de pandemia y las consecuencias del aislamiento social para los mayores. El envejecimiento saludable es necesario en la actualidad, ya que esta población corresponde a la mayor porción vulnerable a las consecuencias de la contaminación por el coronavirus. Conclusión: Por lo tanto, son necesarias intervenciones sobre la salud de los adultos mayores en el período pandémico, con el fin de brindar opciones para la continuidad de la calidad de vida. Palabras-clave: aislamiento social, calidad de vida, anciano, pandemia, impacto social.

Introdução

No final do ano de 2019, houve um surto de uma nova doença respiratória detectada na cidade de Wuhan, na China, provocado pelo novo coronavírus intitulado COVID-19. Em aproximadamente dois meses foram detectados milhares de casos, resultando em inúmeros óbitos. Anteriormente, aconteceram outras duas epidemias de coronavírus chamadas de Síndrome Respiratória Aguda Grave (SARS) e Síndrome Respiratória do Oriente Médio (MERS), mas a COVID-19 se destaca pela rapidez de disseminação e pelas dificuldades para contenção. Portanto, em março de 2020 a Organização Mundial da Saúde (OMS) declarou a ocorrência de uma pandemia pelo novo coronavírus [1].

Muito se é discutido no atual contexto social, medidas que promovem maior segurança quando se fala de saúde pública e qualidade de vida. Segundo o Ministério da Saúde (MS), durante a presente pandemia, é necessária responsabilidade, tendo em vista que seu contágio é facilitado pelo contato próximo. Assim, faz-se necessário evitar aglomerações, preservando a população de modo geral e a de maior risco [2].

Neste contexto, pode-se abordar o distanciamento social, que consiste em evitar o contato próximo entre as pessoas e sendo recomendado o distanciamento físico de no mínimo um metro e meio. Já o isolamento social ocorre quando o número de contágios de determinada patologia ultrapassa os valores previstos em determinado local e tempo. Durante o período de quarentena, a população é orientada a evitar sair de casa, e caso tenham suspeitas, é necessário o isolamento físico por no mínimo quatorze dias, por ser o período de incubação do atual agente infeccioso [3].

A história mostra que esta não é a primeira vez que a sociedade recorre a uma conduta de isolamento. No estado de São Paulo, em dezembro de 1923, medidas similares foram aplicadas para controle do surto de hanseníase, porém de forma mais incisiva e segregativa. Com o intuito profilático, os sintomáticos eram alocados em asilos, sanatórios e colônias agrícolas que eram vulgarmente nomeadas de "vilas de leprosos", podendo ter anexos como orfanatos, creches, asilos e hospitais [4].

Partindo do mesmo princípio profilático, porém com uma abordagem humanizada e com embasamento científico, a restrição social apresentada na atualidade devido à disseminação do coronavírus (SARS CoV-2) funciona de forma a proteger a população, tanto de forma singular como coletivamente, informando sobre riscos e conscientizando quanto às abordagens preventivas. Evita-se, assim, aglomerações e reduz-se a propagação do vírus [5].

O grupo de risco no presente momento consiste principalmente nos idosos, pessoas com patologias crônicas e imunocomprometidos, não deixando de lado os grupos que são restritos a ambientes fixos, como a população privada de liberdade, imigrantes que transitam pela alfândega e residentes de Instituições de Longa Permanência (ILPs) [6]. 
De acordo com a OMS, a população idosa tem maior suscetibilidade para contrair a COVID-19, pois os quadros se agravam em uma maior velocidade, por consequência, geram taxas mais elevadas de mortalidade [7]. Segundo o MS, o maior quantitativo de óbitos notificados até junho de 2020 no Brasil corresponde à população com faixa etária acima dos 60 anos, com 53.223 óbitos, equivalente a 70\%, sendo em sua maioria do sexo masculino e de cor parda [1].

Para o enfrentamento da pandemia pelo novo coronavírus, uma das estratégias é 0 isolamento de pessoas doentes pela COVID-19 por representarem risco de contágio a outras pessoas; dessa forma, é crucial afastá-las do convívio social. Tal prática envolve medidas de distanciamento social também entre as pessoas aparentemente sadias, para garantir a saúde da população e impedir o colapso do sistema de saúde [8].

$\mathrm{O}$ isolamento social tem sido adotado por vários países durante a pandemia na tentativa de achatar a curva de contágio da doença, mas não se pode esquecer que a população idosa, mais especificamente portadores de doenças crônicas, têm maior suscetibilidade ao contágio do vírus e maior vulnerabilidade $[9,10]$.

Durante o período de quarentena, o direito de equidade dos idosos deve ser reforçado. Deve-se zelar por estes indivíduos conforme suas necessidades, para que seja mantida mesmo em momentos de crise. Pensando nisto, durante o isolamento social diversas experiências podem reduzir a qualidade de vida dessa população, sendo necessária maior abordagem sobre o tema do presente trabalho [11].

O presente estudo objetivou apresentar o impacto do isolamento social na vida da pessoa idosa na atual pandemia por COVID-19.

\section{Material e métodos}

Trata-se de uma revisão de literatura narrativa. $O$ acervo bibliográfico acessado foi obtido através das seguintes bases de dados eletrônicos: Scientific Electronic Library Online (Scielo), Literatura Latino-Americana e do Caribe de Informações em Ciências da Saúde (Lilacs) e Sistema de Publicação Eletrônica de Teses e Dissertações (TEDE). Obtém-se, por meio destas bases, artigos e revistas científicas publicados em português e inglês. A busca ocorreu em junho de 2020 com utilização das palavras-chave "isolamento social", "distanciamento social", "idosos", "qualidade de vida" e "pandemia".

A pesquisa narrativa permite ao leitor entender a experiência do pesquisador; trata-se de um estudo que evidencia pontos que outros autores já abordaram e práticas de campo já realizadas. Quando se é falado sobre este tipo de estudo, o pesquisador necessita interpretar artigos e materiais científicos para assim transcrever uma perspectiva acadêmica e profissional. Os dados da pesquisa são então coletados de forma qualitativa e, posteriormente, o pesquisador decide o perfil que mais se adequa ao seu estudo [12,13].

Foram utilizados os seguintes descritores, adquiridos na plataforma "Descritores em Ciência da Saúde (DeCS)": isolamento social; qualidade de vida; idoso; pandemia; impacto social.

O recorte temporal utilizado para a seleção dos conteúdos deste trabalho foi entre 2000 e 2020, com o intuito de captar conteúdos para maior obtenção de dados relevantes sobre o propósito do estudo.

Resultados

É fundamental ressaltar que ser idoso não está associado a perder qualidade de vida, muito pelo contrário. $O$ "envelhecer" é exemplificado através de duas vertentes, uma com baixa qualidade de vida e outra de alta qualidade de vida, já que muitos fatores podem tanto afastar quanto aproximar esses dois extremos [14]. A longevidade da população é o produto do desenvolvimento da sociedade, visto que o idoso do século 21 é mais propenso a doenças crônicas; mas por outro lado, também possui melhor e maior acesso à informação, advinda de familiares, conhecidos, ou mesmo oriunda do Sistema Único de Saúde (SUS) [15].

\section{Qualidade de vida e a senescência}

Muito se é discutido quando o assunto é qualidade de vida do idoso. Assim sendo, para que esse tema seja abordado é necessário ter entendimento a respeito do envelhecimento, que não deve ser visto como uma experiência homogênea. Partindo do ponto que cada pessoa possui um ponto de vista diferente, variando de acordo com seus costumes, crenças, princípios 
e vivências. Sabendo-se que cada idoso tem uma vivência única, a qualidade de vida requer um limiar mínimo aceitável para que exista uma classificação, sendo alguns desses elementos o direito à saúde, ao saneamento básico, à moradia e à mobilidade; acesso ao lazer, à boa alimentação e à água tratada e, principalmente, o direito à dignidade e ao respeito, exercendo assim sua cidadania [11-15].

A prática de exercício físico é o maior fator protetor para os agravos do envelhecimento. Essa prática de forma regular reduz as taxas de morbimortalidade, assim como uma maior expectativa de vida quando comparados a pessoas que não praticam. Em sequência, é evidenciado que a medida não farmacológica de primeira escolha para a prevenção e tratamento de diversas patologias é a atividade física constante, principalmente para a qualidade de vida de pacientes crônicos, como os hipertensos, diabéticos, obesos entre outros [16].

Para o idoso, a alimentação saudável está entre os principais cuidados a serem realizados. Com o passar das décadas, o metabolismo humano tende a reduzir sua atividade de forma gradual. Devido à redução das atividades realizadas, o corpo passa a necessitar de menos energia [17]. Como consequência dessa redução metabólica, é indispensável que o idoso se alimente de forma mais consciente, trocando quantidade por qualidade, ingerindo alimentos ricos em micronutrientes (minerais e vitaminas) presentes em frutas, legumes e verduras em meio a suas refeições. Essa adesão é necessária para manter-se saudável e reduzir os déficits nutricionais esperados do processo de envelhecimento [18].

As consequências de uma alimentação desbalanceada com consumo de alimentos ultraprocessados pode reduzir a expectativa de vida da terceira idade, levando em consideração que hipertensos tendem a possuir uma dieta rica em sódio e diabéticos tendem a preferir consumir alimentos com elevado teor de açúcar. Esses fatores reforçam a necessidade do acompanhamento nutricional para o público geriátrico, visando o desenvolvimento da qualidade de vida, prevenindo e controlando os agravos provenientes das patologias crônicas [19-20].

Relacionado à cidadania, é indispensável para a saúde do idoso conviver em sociedade. Nesta perspectiva mulheres e idosos mais velhos têm maior suscetibilidade para sensação de solidão, assim como pensamentos depressivos. Desta forma, tornam-se necessárias tomadas de decisão por parte dos profissionais e familiares responsáveis, proporcionando os devidos estímulos ao idoso, como, por exemplo, interação com os jovens e com outros idosos de forma segura, entre outras experiências que favoreçam a autonomia desse indivíduo [15].

Para que os jovens idosos possuam "um envelhecimento bem-sucedido", vê-se necessário um investimento em saúde, tanto física quanto mental, reduzindo as chances de contrair ou agravar determinadas patologias, assim como retardar as complicações provenientes do envelhecimento [21].

\section{Isolamento social dos idosos em tempos de pandemia}

Considerando as dificuldades enfrentadas pela população idosa, durante esse período de transição etária, diversas alterações sociais ocorrem. Dentre elas, destaca-se o evento da aposentadoria, que deveria ser o momento de descanso e reconhecimento por sua longa jornada, mas que, por vezes, torna-se o período no qual o indivíduo deixa de exercer um papel claro na sociedade [14].

Deve-se considerar também que ocasionalmente esses idosos vivem o luto por seus companheiros, parentes e conhecidos. Como resultado essa população acaba desenvolvendo uma rotina menos ativa e aos poucos mais dependentes de seus familiares, perdendo a tão necessária autonomia. Esses fatores físicos e emocionais presentes no cotidiano tendem a reduzir a qualidade de vida desse grupo de risco [22].

Durante o período pandêmico de 2020, ao grupo de risco acima de 65 anos é recomendado um isolamento social mais rigoroso, realizado não por escolha, mas como método preventivo a exposição e contágio do vírus. O distanciamento do convívio com a comunidade é tolerado de forma diferente pelos grupos de idosos que possuem companhia, seja ela de familiares, cônjuges ou amigos, lidando com as consequências desse isolamento de forma mais branda. No entanto, idosos que vivem sozinhos ou em ILPs, que têm seu contato reduzido com familiares ou mesmo com a sociedade, sofrem as consequências do distanciamento social de forma mais intensa [23-24].

Após a aposentadoria há a comum sensação de não pertencimento ao local, já que exerceu em algum momento um forte papel de trabalhador dentro da sociedade e suas experiências eram relevantes nesse contexto. Todavia, no ambiente doméstico, essas vivências 
não são significativas para manter a diálogo constante, reduzindo assim a comunicação entre o grupo familiar [25].

A comunicação é sem dúvida o aspecto mais abordado dentre as discussões relativas à melhoria da qualidade de vida no isolamento social. Por conseguinte, durante a pandemia, o fator digital tem ganhado espaço, pois jovens e idosos tendem a passar mais tempo ociosos, preservando por meio online a integridade desse grupo de risco. Idosos que têm acesso ao ensino digital apresentaram maior progresso em controle de movimentos finos, performance cognitiva, linguística e redução dos sintomas de depressão, quando contraposto ao grupo controle $[26,27]$.

Ao observar o confinamento das pessoas no contexto atual, deve-se também ser considerada a influência e a toxicidade das mídias digitais acerca da população idosa, de forma que esses indivíduos que passam grande parte do dia diante da televisão, ou de celulares, vivenciam o assunto coronavírus ser saturado ao longo do dia [28].

Há dois posicionamentos extremos prevalentes que são observados, sendo o primeiro relacionado ao otimismo irreal que é norteado por "no fim vai dar tudo certo independente das suas ações", subestimando assim as consequências, e podendo esse indivíduo se expor de forma imprudente, quebrando o distanciamento social. Já o segundo consiste em observar de forma distorcida e negativa, bem como adotar medidas de preocupação intensa e por vezes associadas ao medo, à angústia e à tristeza. Enquanto o primeiro grupo tem mínimas precauções, o segundo ultrapassa os limites comprando de forma exacerbada recursos de proteção e higiene, afetando assim o mercado e o ambiente profissional, que por vezes não possuem recursos mínimos para atendimento do público que necessita [29].

Neste contexto, observa-se a importância do grupo de apoio a esses idosos nos momentos de dificuldade decorrentes do isolamento social profilático, sendo estes tanto profissionais quanto familiares. Devem ser elaboradas estratégias para inclusão do indivíduo dentro do ambiente doméstico, tornando-o parte da rotina e assim melhorando sua qualidade de vida $[30,31]$.

\section{As consequências do isolamento social para o idoso}

Ao longo do trabalho foi apresentado o perfil heterogêneo da população idosa perante o atual contexto social. Nessa perspectiva, o envelhecimento traz diversas alterações fisiológicas que por vezes se mostram nítidas em sua rotina, nas alterações de humor, no sedentarismo e na alimentação, influenciando diretamente na qualidade de vida desses indivíduos, que passam a sentir de forma mais intensa as alterações em seu cotidiano, tanto de forma social quanto pessoal [11].

Após o fim do surto causado pela COVID-19, será possível visualizar os danos psicológicos causados na população como consequência do atual cenário, mostrando que esse desgaste mental pode ser comparado aos mesmos sentimentos provenientes de catástrofes naturais e cenários de guerra pelos quais a sociedade passa por estresse, tensão, ansiedade, frustração, insegurança relacionada ao futuro e pelo medo da morte [32].

Em razão do desenvolvimento dessas emoções negativas, houve um aumento importante de novos casos de transtornos psicológicos, tornando a população que não possui uma rede de apoio presente, durante e após o isolamento social, mais suscetíveis a estes transtornos. Dentre esses, merecem atenção os associados ao trauma, como o transtorno de estresse pós-traumático e os transtornos depressivos, provenientes do longo período de isolamento, associado à grande quantidade de informações negativas acompanhadas por essa população, durante o período de quarentena, tornando a rotina opressiva e frustrante, causando cansaço tanto físico quanto emocional [28].

Uma clara alteração na sociedade pós-pandemia poderá ser a diminuição do contato físico de forma geral, características sociais já difundidas na sociedade oriental [33]. Os idosos poderão sentir maior impacto com essas alterações sociais por estarem mais próximos dos familiares e amigos [34].

Como consequência das medidas de segurança através do isolamento social, a população idosa que antes praticava atividades ao ar livre passa a sair cada vez menos de suas casas, por vezes priorizando a segurança e por outras por medo do desconhecido. Portanto, esses fatores estabelecem uma situação complexa, tanto psicologicamente quanto fisicamente, pois os idosos precisam manter o corpo ativo [16].

Muito se discute sobre os benefícios da vitamina $D$ no organismo, ainda mais se tratando da população idosa que, devido ao processo de alterações anatomofisiológicas, encontra-se 
imunodeprimida. Porém, durante o isolamento social, esse grupo sai cada vez menos de casa, deixando de lado a necessidade de exposição à luz solar, que é a principal responsável pela síntese desse pró-hormônio que associado ao paratormônio (PTH) atua como importante regulador do metabolismo ósseo. Estudos atuais mostram sua eficácia em tratamentos de idosos com depressão, assim como sua importância relacionada ao metabolismo e efeitos protetores nos sistemas esquelético, muscular e imunológico [35].

Um dos principais agravos causados pelo distanciamento social é a diminuição da procura por suporte de saúde, retardando esse contato até o momento mais crítico, uma vez que ambientes hospitalares e ambulatoriais são locais de grande movimentação de pessoas enfermas e possivelmente contaminadas com o coronavírus. Visando estes fatores, tanto para diminuir as demandas hospitalares, como para garantir maior segurança da população de risco, as instituições de saúde estão promovendo atendimentos on-line [36].

Outro fator existente são as alterações associadas ao padrão de sono, que são comumente relacionadas a disfunções psicogênicas. A qualidade do sono está diretamente ligada à qualidade de vida, sendo compreensível que idosos que relatam distúrbios do sono tenham maior vulnerabilidade, muitas vezes associada a problemas de saúde como depressão, instabilidade emocional, distorcida percepção da saúde e presença de doenças crônicas [37].

\section{Conclusão}

Conclui-se que o envelhecimento saudável se faz necessário no atual momento, pois essa população corresponde à maior parcela vulnerável às consequências da contaminação pelo coronavírus, e fatores que proporcionam a melhora na qualidade de vida são considerados fatores protetores a saúde desses idosos.

Pode se observar que o idoso é naturalmente suscetível ao isolamento social, mesmo em momentos de homeostase social. Portanto, quando exposto a um fator estressante como a atual pandemia, são necessários maiores investimentos em cuidados preventivos capazes de garantir a segurança e reduzir os danos causados a esse grupo de risco.

Vê-se necessária a intervenção quanto às medidas de atuação a respeito da saúde do idoso no período pandêmico, fornecendo opções da continuidade da qualidade de vida e possibilidades de comunicações seguras com a rede de apoio à pessoa idosa, tendo a tecnologia como seu maior aliado. Igualmente, nota-se a necessidade de tomadas de decisão por parte do governo, relacionadas à melhora da qualidade de vida de forma efetiva da população idosa no momento pós-pandemia.

Fatores que envolvem a desigualdade social não foram abordados no presente trabalho por serem imprevisíveis as futuras alterações socioeconômicas, já que estamos em meio ao processo pandêmico.

Frente ao exposto, conclui-se que apesar do objetivo proposto quanto ao impacto do isolamento social para os idosos ter sido alcançado. Por estar vigente o período de pandemia, acredita-se que existe uma estreita relação entre os impactos provenientes do isolamento social aos idosos com a atual situação de isolamento social em virtude da COVID-19. São necessários mais estudos que tragam estimativas conclusivas a respeito do impacto da pandemia sobre a população de idosos. Considerando a escassez das informações, sugere-se que sejam realizadas mais pesquisas a fim de trazer a completude necessária sobre a referida temática.

1. Brasil. Ministério da Saúde. O que é coronavírus? (COVID-19). [citado 2020 Jun 6]. Disponível em: https://coronavirus.saude.gov.br/

2. Brasil. Ministério da Saúde. Como é definido um caso suspeito de coronavírus? [citado 2020 Jun 6]. Disponível em: https://coronavirus.saude.gov.br/sobre-adoenca\#tratamento

3. Brasil. Ministério da Saúde. Secretaria de Atenção Especializada à Saúde. Departamento de Atenção Hospitalar, Domiciliar e de Urgência. Protocolo de manejo clínico da COVID-19 na Atenção Especializada / Ministério da Saúde, Secretaria de Atenção Especializada à Saúde, Departamento de Atenção Hospitalar, Domiciliar e de Urgência. - 1. ed. rev. - Brasília, 2020. [citado 2020 Jun 14]. Disponível em: https://bvsms.saude.gov.br/bvs/publicacoes/manejo clinico COVID19 atencao especializada.pdf 
4. Silva EG, Romero M. O controle da hanseníase em São Paulo: Departamento de Profilaxia da Lepra (1930-1969) [Tese]. São Paulo: Pontifícia Universidade Católica de São Paulo; 2018. 118f. [citado 2020 Jul 3]. Disponível em:

https://tede.pucsp.br/handle/handle/21492

5. Netto RGF, Corrêa JW. Epidemiologia do surto de doença por coronavírus (COVID-19). Desafios-Rev Interdis Uni Fed Toc 2020;7(Especial3):18-25. https://doi.org/10.20873/uftsuple2020-8710

6. Lemos P, Almeida-Filho N, Firmo J. COVID-19, desastre do sistema de saúde no presente e tragédia da economia em um futuro bem próximo. Brazilian Journal of Implantology and Health Sciences 2020;2(4):39-50. https://doi.org/10.36557/2674$8169.2020 \mathrm{v} 2 \mathrm{n} 4 \mathrm{p} 39-50$

7. Neri M. Onde estão os idosos? Conhecimento contra o COVID-19. FGV Social, abril 2020. [citado 2020 Jul 10]. Disponível em: https://www.cps.fgv.br/cps/bd/docs/SumarioExecutivo-COVIDage-FGV-Social-Marcelo-Neri.pdf

8. CRF-SE (Conselho Regional de Farmácia de Sergipe). Necessidade do isolamento social durante a pandemia. CRF/SE, 2020. [citado 2020 maio 18]. Disponível em: http://crfse.org.br/noticia/1090/crf-se-reforca-a-necessidade-do-isolamento-socialdurante-a-pandemia-do-COVID-19

9. Baker MG, Peckham TK, Seixas NS. Estimating the burden of United States workers exposed to infection or disease: a key factor in containing risk of COVID-19 infection. PLoS One 15(4): e0232452. https://doi.org/10.1371/journal.pone.0232452

10. Filo JMJ, Assunção AA, Algranti E, Garcia EG, Saito CA, Maeno M. A saúde do trabalhador e o enfrentamento da COVID-19. Rev Bras Saúde Ocup 2020;45e14. https://doi.org/10.1590/2317-6369ed0000120

11. Saraiva F, Simões MR, Firmino H. Avaliação psicológica dos comportamentos de condução de pessoas mais velhas. Saúde Mental das Pessoas mais Velhas 2016;183200. [citado 2020 Jun 13]. Disponível em:

https://eg.uc.pt/bitstream/10316/47534/1/Capitulo-lidel.pdf

12. Clandinin DJ, Connelly FM. Narrative inquiry: experience and story in qualitative research. San Francisco: Jossey-Bass, 2000. [citado 2020 maio 8]. Disponível em: http://cae.americananthro.org/wpcontent/uploads/2014/09/Clandinin Jean Connelly Michael-Narrative Inquiry.pdf

13. Sahagoff AP. Pesquisa Narrativa: uma metodologia para compreender a experiência humana. Semana de Extensão, Pesquisa e Pós-Graduação, 2015; v. 11. [citado 2020 Jun 17]. Disponível em: https://www.uniritter.edu.br/files/sepesq/arquivos trabalhos/3612/879/1013.pdf

14. Paschoal SMP. Qualidade de Vida na Velhice. In: Freitas EV, Py L, Neri AL, Cançado FAX, Gorzoni ML., Rocha SM, editores. Tratado de Geriatria e Gerontologia. Rio de Janeiro: Guanabara Koogan; 2002; 79-84.

15. Oliveira LM, Abrantes GG, Ribeiro GS, Cunha NM, Pontes MLF, Vasconcelos. Solidão na senescência e sua relação com sintomas depressivos: revisão integrativa. Rev Bras Geriat Gerontol 2019;22(6)e190241. https://doi.org/10.1590/1981-22562019022.190241

16. Zanesco A, Zaros PR. Exercício físico e menopausa. Rev Bras Ginecol Obst 2009;31(5):254-61. https://doi.org/10.1590/S0100-72032009000500009

17. Andreotti DG, Veiga MLR, Moraes MMG, Begnami MFD, Cogliatti WNJ, Pereira RCG. Fatores nutricionais e físicos que interferem na qualidade de vida do idoso. Rev Bras Multidis 2013;16(2):27-35. https://doi.org/10.25061/2527-2675/ReBraM/2013.v16i2.61

18. Neto FT, Cunha SFC, Cunha DF. Nutrição em geriatria. In: Teixeira Neto F, ed. Nutrição clínica. Rio de Janeiro: Guanabara Koogan; 2003. p.279-290.

19. Previato HDRA et al. Perfil clínico-nutricional e consumo alimentar de idosos do programa terceira idade, Ouro Preto MG. Demetra: Alimentação, Nutrição \& Saúde 2007;10(2):375-87. https://doi.org/10.12957/demetra.2015.15014

20. Alves LC, Leimann BCQ, Vasconcelos MEL, Carvalho MS, Vasconcelos AGG, Fonseca TCO. A influência das doenças crônicas na capacidade funcional dos idosos do Município de São Paulo, Brasil. Cad Saúde Pública 2007;23(8):1924-30. https://doi.org/10.1590/s0102-311x2007000800019

21. Netto MP. Estudo da velhice: histórico, definição do campo e termos básicos. In: Freitas EV, PY L. (Orgs.). Tratado de geriatria e gerontologia. Rio de Janeiro: Guanabara Koogan; 2016. p.3-13. 
22. Farinasso A, Labate R. A vivência do luto em viúvas idosas. SMAD Rev Eletrônic. Saúde Mental Álcool e Drogas (Edição em português) 2015;11(1):25-32. https://doi.org/10.11606/issn.1806-6976.v11i1p25-32

23. Santos PA, Moraes-Filho JP. A percepção do idoso sobre a comunicação no processo de envelhecimento. Audiology - Communication Research 2019; 24,e2058. https://doi.org/10.1590/2317-6431-2018-2058

24. Vechia ADRD, Mamani ARN, Azevedo RCS, Reiners AAO, Pauletto TT, Segri NJ. Tensão do papel de cuidador em cuidadores informais de idosos. Texto e Contexto Enferm 2019;28,e20180197. https://doi.org/10.1590/1980-265x-tce-2018-0197

25. Faro A, Bahiano MA, Nakano TC, Reis C, Silva BFP, Vitti LS. COVID-19 e saúde mental: a emergência do cuidado. Estudos de Psicologia 2020;37:e200074. https://doi.org/10.1590/1982-0275202037e200074

26. Alvarenga GMO, Yassuda MS, Cachioni M. Inclusão digital com tablets entre idosos: metodologia e impacto cognitivo. Psicologia, Saúde \& Doenças, Lisboa 2019;20(2):384401.https://doi.org/10.15309/19psd200209

27. Ferreira S, Torres A, Mealla A, Veloso A. Training effects on older adults in information and communication technologies considering psychosocial variables. Ed Geront 2015;41(7):482-93. https://doi.org/10.1080/03601277.2014.994351

28. Brooks SK, Webster RK, Smith LE, Woodland L, Wessely S, Greenberg N. The psychological impact of quarantine and how to reduce it: rapid review of the evidence. Lancet 2020;395(10227):912-20. https://doi.org/10.1016/S0140-6736(20)30460-8

29. Raude J, Debin M, Souty C, Guerrisi C, Turbelin C, Falchi A et al. Are people excessively pessimistic about the risk of coronavirus infection? PsyArXiv Preprints 2020. https://doi.org/10.31234/osf.io/364ai

30. Nascimento HG, Figueiredo AEB. Demência, familiares cuidadores e serviços de saúde: o cuidado de si e do outro. Ciênc Saúde Coletiva 2019;24(4):1381-92. https://.doi.org/10.1590/1413-81232018244.01212019

31. Labegalini CMG et al. Percurso cuidativo-educativo dialógico sobre envelhecimento ativo com profissionais da estratégia saúde da família. Texto Contexto Enferm 2020;29,e20180235. https://doi.org/10.1590/1980-265x-tce-2018-0235

32. Fiorillo A, Gorwood P. The consequences of the COVID-19 pandemic on mental health and implications for clinical practice. Eur Psych 2020;63(1). https://doi.org/10.1192/j.eurpsy.2020.35

33. Braun J. Os cumprimentos e contatos físicos não serão como antes: Trata-se de um sacrifício para sociedades cordiais. Veja, n 2694. Abril; 3 jul. 2020. [citado 2020 Jul 12]. Disponível em: https://veja.abril.com.br/ideias/os-cumprimentos-e-contatos-fisicos-naoserao-como-antes/

34. Suvilehto JT, Glerean E, Dunbar RIM, Hari R, Nummenmaaet L. Topography of social touching depends on emotional bonds between humans. PNAS 2015;112(45):1381116. https://doi.org/10.1073/pnas.1519231112

35. Williams J, Williams C. Responsibility for vitamin D supplementation of elderly care home residents in England: falling through the gap between medicine and food. MedRxiv 2020. https://doi.org/10.1101/2020.06.21.20136697

36. Vidal GP, Castro A. O Psicodrama clínico on-line: uma conexão possível. Rev Bras Psicodrama 2020;28(1):54-64. [citado 2020 Jul 21]. Disponível em: http://pepsic.bvsalud.org/scielo.php?script=sci arttext\&pid=S0104$53932020000100006 \&$ Ing $=$ pt\&nrm $=$ iso

37. Lopes JM, Roncalli AG. Fatores biopsicossociais associados à função de percepção do sono em idosos brasileiros: análise de uma pesquisa nacional. Rev Bras Epidemiol 2020;23,e200083. https://doi.org/10.1590/1980-549720200083 